\title{
Non-Born-Oppenheimer calculations of the HD molecule in a strong magnetic field
}

\author{
Ludwik Adamowicz ${ }^{1}{ }^{*}$ and Erik I. Tellgren ${ }^{2}$, and Trygve Helgaker ${ }^{2}$ \\ ${ }^{1}$ Department of Chemistry and Biochemistry, University of Arizona, Tucson, Arizona 85721, USA \\ ${ }^{2}$ Centre for Theoretical and Computational Chemistry (CTCC), Department of Chemistry, \\ University of Oslo, P.O.Box 1033 Blindern, N-0315 Oslo, Norway
}

An effective variational non-Born-Oppenheimer method is applied to calculate the ground state of the HD molecule in a strong magnetic field. The Hamiltonian used in the calculations is obtained by subtracting the operator representing the kinetic energy of the center-of-mass motion from the total laboratory-frame Hamiltonian. Orbital basis sets are used for the deuteron, the proton, and the electrons. Based on the calculated expectation values, it is determined that, with increasing field strength, the bond length decreases and the alignment of the molecule with the field increases.

\section{INTRODUCTION}

The interaction of atoms and molecules with external magnetic fields, including very strong fields, has been intensely studied for the last three decades [1-13]. The conventional approach for calculating ground and excited bound states of atoms and molecules placed in a uniform magnetic field without the Born-Oppenheimer (BO) approximation involves the separation of the center of mass motion. This is usually done by selecting a coordinate system in which the Hamiltonian automatically separates into an operator representing the kinetic energy of the center-of-mass motion and an internal Hamiltonian representing the system's internal state. There have been several works concerning the separation of the center-of-mass motion for molecular systems (mostly diatomic systems) interacting with a uniform magnetic field [14-22]. The properties of the total pseudo-momentum of the system expressed in terms of relative coordinates were exploited in those works to partition the resulting Hamiltonian operator into an electronic part and a nuclear part. Although the magnetic-field modification of the nuclear Hamiltonian and the electronic-nuclear coupling have not been investigated in detail, there have been reports on the validity of the BO approximation for a hydrogen

*Electronic address: ludwik@email.arizona.edu 
atom [15] and a diatomic molecule [16, 20], and on how the magnetic field affects the rotations and vibrations of neutral diatomic molecules [21]. To our knowledge, there have been no practical applications of the direct non-BO approach in calculations of ground and excited states of molecular systems in the presence of a magnetic field.

Here we present an alternative non-BO method for describing the behavior of a molecule in a magnetic field. The Hamiltonian operator used in the approach is obtained by subtracting the operator representing the motion of the center of mass expressed in the Cartesian laboratory coordinate system from the total non-relativistic Hamiltonian expressed in this coordinate system. The approach is implemented and used in model calculations of the HD molecule.

\section{THE METHOD}

In the present calculations, we employ the effective non-Born-Oppenheimer (non-BO) method introduced by Kozlowski and Adamowicz [23]. The starting point is the total nonrelativistic Hamiltonian of the system without a magnetic field written in terms of the laboratory Cartesian coordinates, $\mathbf{R}_{l}, l=1, \ldots, N$, where $N$ is the number of particles forming the system (the number of the nuclei plus the number of the electrons). In atomic units, the Hamiltonian is:

$$
\hat{H}=-\sum_{l}^{N} \frac{1}{2 M_{l}} \nabla_{\mathbf{R}_{l}}^{2}+\sum_{k>l}^{N} \frac{Q_{k} Q_{l}}{R_{k l}} .
$$

In (1), $M_{l}$ and $Q_{l}$ are the mass and the charge of particle $l$, respectively, and $R_{k l}$ is the distance between particle $k$ and particle $l$. In the next step, the coordinates of the center of mass of the system are defined:

$$
\mathbf{R}_{c m}=\frac{\sum_{l}^{N} M_{l} \mathbf{R}_{i}}{M},
$$

where $M=\sum_{l}^{N} M_{l}$ is the total mass of the system, and used to write the operator, $\hat{T}_{c m}$, representing the kinetic energy of the center-of-mass motion:

$$
\hat{T}_{c m}=-\frac{1}{2 M} \nabla_{\mathbf{R}_{c m}}^{2}
$$

The effective Hamiltonian representing the internal energy of the system, $\hat{H}_{i n t}$, is the difference between the laboratoryframe Hamiltonian, $\hat{H}$ and $\hat{T}_{c m}$ :

$$
\hat{H}_{i n t}=\hat{H}-\hat{T}_{c m}
$$

Note that, as $\hat{T}_{c m}=\frac{1}{2 M} \hat{P}_{c m}^{2}$, where $\hat{P}_{c m}$ is the canonical momentum operator associated with the motion of the center of mass,

$$
\hat{T}_{c m}=\frac{\left(\sum_{l}^{N} \hat{\mathbf{p}}_{l}\right)^{2}}{2 M}
$$


where $\hat{\mathbf{p}}_{l}$ is the operator representing the canonical momentum of particle $l . \hat{H}_{\text {int }}$ is used in the calculation of the internal bound states of the system.

As one notices, in the effective non-BO method, a separation of the laboratory-frame total Hamiltonian into an internal Hamiltonian and a center-of-mass kinetic-energy Hamiltonian is not explicitly performed. Instead, an effective internal Hamiltonian is constructed as is the difference of the former and the latter. Note that the effective internal Hamiltonian depends on the laboratory coordinates of all particles forming the system. The wave function therefore also depends on these coordinates.

The wave function in the effective non-BO method, which depends on the laboratory coordinates of the particles (nuclei and electrons) forming the system and on their spins, has to be properly symmetrized (for bosons) and antisymmetrized (for fermions) with respect to the permutations within each group of identical particles. For example, the wave function representing the $\mathrm{HD}$ molecule has to be antisymmetric with respect to the permutation of the electron labels. Moreover, the wave function representing the ground state has to be a product of a spatial function that is symmetric with respect to the permutation of the electrons and a spin function that is an antisymmetric function with respect to this permutation. The spatial wave function representing the ground-state HD molecule is: $(1+\hat{P}(1,2)) \Psi\left(\mathbf{R}_{d}, \mathbf{R}_{p}, \mathbf{R}_{1}, \mathbf{R}_{2}\right)$, where indices $d, p, 1$, and 2 indicate the deuteron, the proton, and the two electrons, respectively. To obtain the total wave function, the above spatial function is multiplied by the product of a spin function for the deuteron, a spin function for the proton, and an antisymmetric spin function for the two electrons.

For high accuracy in non-BO calculations of bound states of a system with Coulombic interactions, the wave function should be expanded in basis functions that explicitly depend on the distances between particles, known as explicitly correlated basis functions $[24,25]$. However, in this first model for non-BO calculations of a molecule in a magnetic field, we use an expansion in products of one-particle functions (orbitals). A similar orbital approach for describing the nuclear motion was used before by, for example, Nakai et al. [26] and Nakatsuji et al. [27]. Three different sets of such functions are used for the HD molecule, the system considered in this initial model application. The first set contain the orbitals, $\psi_{i}^{d}$, used to expand the component of the total wave function describing the state of the deuteron, the second set contain the orbitals, $\psi_{i}^{p}$, describing the state of the proton, and the third set contain the orbitals, $\psi_{i}^{e}$, describing the state of the two electrons. Thus, the total spatial ground-state wave function for the HD molecule is a superposition of products of the orbitals belonging to the three sets:

$$
(1+\hat{P}(1,2)) \Psi\left(\mathbf{R}_{d}, \mathbf{R}_{p}, \mathbf{R}_{1}, \mathbf{R}_{2}\right)=\sum_{i} \sum_{j} \sum_{k \geq l} c_{i j k l} \psi_{i}^{d}\left(\mathbf{R}_{d}\right) \psi_{j}^{p}\left(\mathbf{R}_{p}\right)(1+\hat{P}(1,2))\left[\psi_{k}^{e}\left(\mathbf{R}_{1}\right) \psi_{l}^{e}\left(\mathbf{R}_{2}\right)\right]
$$

where $c_{i j k l}$ are the linear expansion coefficients. 
We now introduce the interaction with a static magnetic field into the effective internal Hamiltonian, $\hat{H}_{i n t}$. In doing this, we distinguish between the canonical momentum operator $\hat{\mathbf{p}}_{l}=-i \hbar \nabla_{l}$ and the gauge-invariant kinetic momentum operator

$$
\hat{\pi}_{l}=-i \hbar \nabla_{l}-Q_{l} \mathbf{A}\left(\mathbf{r}_{l}\right)
$$

where $Q_{l}$ is the charge of particle $l$. The magnetic vector potential $\mathbf{A}(\mathbf{r})$ represents an external magnetic field $\mathbf{B}=\nabla \times \mathbf{A}(\mathbf{r})$. In the present work, we restrict attention to uniform magnetic fields and vector potentials of the form

$$
\mathbf{A}\left(\mathbf{r}_{l}\right)=\frac{1}{2} \mathbf{B} \times\left(\mathbf{r}_{l}-\mathbf{g}\right)
$$

where $\mathbf{g}$ is the gauge origin. The total kinetic energy operator in the presence of a static magnetic field is then given by

$$
\hat{T}=\sum_{l}^{N} \frac{\hat{\pi}_{l}^{2}}{2 M_{l}}=\sum_{l}^{N}\left[-\hbar^{2} \nabla_{l}^{2}-\mathrm{i} \hbar q_{l} \mathbf{B} \cdot\left(\left(\mathbf{r}_{l}-\mathbf{g}\right) \times \nabla_{l}\right)+q_{l}^{2}\left|\mathbf{A}\left(\mathbf{r}_{l}\right)\right|^{2}\right] / 2 M_{l},
$$

where we have omitted the spin Zeeman interactions. The second term in the parenthesis can be identified as $\mathbf{B} \cdot \hat{\mathbf{L}}_{\mathbf{g} ; l}$, with $\hat{\mathbf{L}}_{\mathbf{g} ; l}=\left(\mathbf{r}_{l}-\mathbf{g}\right) \times \hat{\mathbf{p}}_{l}$ denoting the canonical momentum relative to $\mathbf{g}$. This term represents the orbital Zeeman effect and vanishes for states with zero angular momentum. For time-reversal symmetric states, represented by realvalued wave functions, this term is zero. The term is also trivial for states that are related to a time-reversal symmetric state by a gauge transformation, which introduces the same single-valued phase factor into all particle coordinates.

Using the vector identity $|\mathbf{u} \times \mathbf{v}|^{2}=u^{2} v^{2}-(\mathbf{u} \cdot \mathbf{v})^{2}$, we obtain for the diamagnetic contribution in the Hamiltonian

$$
q_{l}^{2}\left|\mathbf{A}\left(\mathbf{r}_{l}\right)\right|^{2}=\frac{q_{l}^{2}}{4} B^{2}\left|\mathbf{r}_{l}-\mathbf{g}\right|^{2}-\frac{q_{l}^{2}}{4}\left[\mathbf{B} \cdot\left(\mathbf{r}_{l}-\mathbf{g}\right)\right]^{2}
$$

Taking the origin of the coordinate system to coincide with the gauge origin and the $z$-axis to coincide with the magnetic field direction, we obtain

$$
\mathbf{g}=0, \quad \mathbf{B}=B \mathbf{e}_{z}=\left(0,0, B_{z}\right)
$$

hence, $B_{x}=B_{y}=0$. Effectively, the particles are then subject to an external potential of the form

$$
V_{l}\left(x_{l}, y_{l}, z_{l}\right)=\frac{q_{l}^{2}}{2 M_{l}}\left|\mathbf{A}\left(\mathbf{r}_{l}\right)\right|^{2}=\frac{q_{l}^{2}}{8 M_{l}} B_{z}^{2}\left(x_{l}^{2}+y_{l}^{2}\right)
$$

where $\mathbf{r}_{l}=\left(x_{l}, y_{l}, z_{l}\right)$. The potential places each particle in a quadratic (harmonic) well that is proportional to the squared particle charge, the squared field strength, and the inverse particle mass. Moreover, the potential acting on particle $l$ depends only on its $x$ and $y$ coordinates, being independent of its $z$ coordinate. The non-BO Hamiltonian 
representing a molecule placed in the magnetic field along the $z$ axis is a sum of $\hat{H}_{\text {int }}$ and $B_{z}^{2} \sum_{l}^{N} \frac{q_{l}^{2}}{8 m_{l}}\left(x_{l}^{2}+y_{l}^{2}\right)$. The orbital Zeeman terms may be omitted because our choice of basis set effectively targets states for which they vanish.

The term $\hat{H}_{\text {int }}$ includes subtraction of the operator representing the kinetic energy of the center-of-mass motion (5). In the presence of the magnetic field, the canonical momentum operators $\hat{p}_{l}$ in $\hat{T}_{c m}$ are replaced by the kinetic momentum operators $\hat{\pi}_{l}(7)$,

$$
\hat{T}_{c m}=\frac{\left(\sum_{l}^{N} \hat{\pi}_{l}\right)^{2}}{2 M} .
$$

In the spirit of the effective character of the present method, we here make an approximation, representing $\hat{T}_{c m}$ in the presence of the field as:

$$
\hat{T}_{c m}=\frac{\hat{\Pi}^{2}}{2 M} \approx \frac{\left[\hat{\mathbf{P}}+Q_{\mathrm{tot}} \mathbf{A}\left(\mathbf{R}_{\mathrm{cm}}\right)\right]^{2}}{2 M}
$$

where $Q_{t o t}=\sum_{l}^{N} Q_{l}$ is the total charge of the system and $\hat{\Pi}=\sum_{l}^{N} \hat{\pi}_{l}$. Since $Q_{t o t}$ is zero for a neutral system, the second term in Eq. (14) vanishes. The approximation in (14) is based on the fact that, classically speaking, the center-of-mass motion of the system in the presence of the magnetic field is a combination of system's translational motion and the motion induced by the interaction of the field with the total charge of the system; the approximation somewhat modifies the coupling between the motions of the individual particles and the center-of-mass motion that appears in the magnetic field [9]. Specifically, a term proportional to the cross product of the magnetic field and total dipole moment is missing in the parenthesis on the right-hand side. In the absence of a field, the coupling vanishes and the total nonrelativistic laboratory-frame Hamiltonian can be rigorously separated into an operator representing the center-of-mass motion and an internal Hamiltonian independent on $\mathbf{R}_{c m}$.

In the absence of a magnetic field, $\hat{H}_{\text {int }}$ is isotropic, i.e., spherically symmetric). Its eigenfunctions are therefore 'atom-like', transforming according to the irreducible representations of the $\mathrm{SO}(3)$ rotation group - in particular, the ground state of HD is represented by a spherically symmetric wave function. When a uniform magnetic field $\mathbf{B}=\left(0,0, B_{z}\right)$ is applied, the rotational motion of the molecule becomes 'polarized'. With increasing field strength, HD becomes increasingly more aligned along the $z$-axis, as its ground-state non-BO wave function becomes more cylindrical.

To describe the cylindrical symmetry in the orbital approximation adopted here (6), the orbitals representing the deuteron, $\psi_{i}^{d}\left(\mathbf{R}_{d}\right)$, the proton, $\psi_{j}^{p}\left(\mathbf{R}_{p}\right)$, and the electrons, $\psi_{k}^{e}\left(\mathbf{R}_{i}\right)$, must have a definite symmetry with respect to rotation about the $z$ axis. The most general such states would acquire phase shifts under small rotations about the $z$ axis and have multivalued phase functions (compare spherical harmonics). The orbitals used in the present calculations 
are spherically symmetric Gaussian functions with centers shifted along the $z$ axis: $g_{k}(\mathbf{r})=\exp \left[-\alpha_{k}\left(\mathbf{r}-\mathbf{s}_{k}\right)^{2}\right]$, where $\mathbf{s}$ is the shift vector (in the HD calculations $s_{k x}=s_{k y}=0$ and $s_{k z} \neq 0$ ). As a consequence, only states of zero canonical angular momentum $\hat{\mathbf{L}}_{\mathbf{g} ; l}$ can be described.

In the present calculations, we use the standard variational method. The ground-state wave function and its energy are obtained by energy minimization with respect to the linear expansion coefficients, $c_{i j k l}$, the orbital exponents, $\alpha_{k}$, and the $z$ coordinates of the Gaussian shift vectors, $\mathbf{s}_{k}$. Since the subtraction of $\hat{T}_{c m}$ in (4) effectively eliminates the translational motion from the description, optimization of the nonlinear parameters of the orbitals representing the deuteron, the proton, and the electrons, does not delocalize the system by 'diffusing' it in space. Such a diffusing would have occurred if the kinetic energy had been part of the energy that is minimized because the minimum energy (zero) would then represent a situation with the center of mass completely delocalized in space.

In presenting the results, we use the notation $(e / p / d)$ to denote the numbers of the electron orbitals $(e)$, the proton orbitals $(p)$, and the deuteron orbitals $(d)$, respectively. The initial values of the orbital exponents are chosen using the STO-3G basis set for the electron in the hydrogen atom, the exponential parameters for the nuclei derived from molecular non-BO calculations performed with explicitly correlated Gaussian functions by the Adamowicz group [28], and the average ground-state HD internuclear distance from the same calculations. In the initial basis set, the proton orbitals are distributed over a relatively short interval of the $z$ axis. The deuteron orbitals are distributed in a similar way such that their location on the $z$ axis is on average equal to the average internuclear distance in the ground vibrational state of the HD molecule. We emphasize that the present calculations are preliminary, being performed to test the possibility of describing a molecule in a static magnetic field without assuming the BO approximation. More accurate calculations with explicitly correlated Gaussians are forthcoming.

\section{RESULTS}

In Table I, the total non-BO energies of HD calculated in a zero magnetic field and in the magnetic field with the strengths of 0.15 and 0.30 atomic units along the $z$-axis are shown, using basis sets ranging from the small set $(6 / 1 / 1 /)$ with a total of 8 orbitals to the larger set $(12 / 4 / 4)$ with 20 orbitals. As the number of orbitals increases, the zero-field energy decreases but is still significantly higher than the non-BO ground-state energy obtained using a large basis of explicitly correlated Gaussians, with the center-of-mass motion explicitly separated from the Hamiltonian by a coordinate transformation [28]. A large number of deuteron, proton, and electron orbitals would be needed to obtain a non-BO energy close to that obtained using explicitly correlated Gaussians or other explicitly correlated functions. 
We note that orbitals aligned along the field direction correctly describe the non-spherical symmetry of the molecule in the presence of a field. In the absence of a field, however, the total molecular non-BO wave function is spherically symmetric and the use of Gaussians distributed along $z$-axis is formally not correct, although the error introduced in this manner is much smaller than other errors introduced in the present calculations.

Even though the non-BO orbital-based wave functions obtained here are highly approximate, the expectation values characterizing the ground state of HD with and without the magnetic field provide interesting insight into the behavior of the molecule in the field, see Table II. The first expectation value is the average deuteron-proton distance. With increasing field strength, the average distance noticeably decreases, as expected from the observation that the confinement potential is proportional to the square of the field strength. The second type of the expectation values shown in Table II corresponds to the average values of the squares of the $x$ coordinates for the proton and the deuteron. As the $x$ axis is perpendicular to the field vector $\mathbf{B}$, the expectation value of $x^{2}$ describes the degree of the alignment of the molecule with the field axis (the $z$ axis). Both $\left\langle x_{d}^{2}\right\rangle$ and $\left\langle x_{p}^{2}\right\rangle$ decrease with increasing field strength, indicating that the molecule becomes increasingly more aligned with the field vector. One point to note is that the $\left\langle x_{d}^{2}\right\rangle$ expectation value is significantly smaller than the value of $\left\langle x_{p}^{2}\right\rangle$ which indicates that the deuteron is more localized in space than the proton. This is an expected behavior resulting from the mass difference of these two particles.

\section{SUMMARY}

An effective method for describing the behavior a neutral molecule in the presence of a magnetic field without assuming the BO approximation is proposed and implemented. The method employs orbitals to represent the states of the nuclei and the electrons forming the molecule. The method is applied to study the non-BO behavior of the HD molecule in the magnetic field. The orbital method only allows for obtaining qualitative results concerning the behavior. The results show that, when the field is turned on, the molecule shrinks along both parallel and perpendicular directions relative to the bond axis and becomes increasingly aligned along the field vector. The parallel shrinking manifests itself in the noticeable decrease of the bond length.

The continuation of this work will involve implementation of explicitly correlated Gaussian functions for describing the behavior of small molecules in a magnetic field with much higher accuracy. As such an implementation involves extended optimization of the non-linear parameters of the Gaussians, the analytical gradient of the field-dependent energy determined with respect to these parameters will be implemented. Moreover, the explicitly correlated Gaussians 
used in the field-dependent implementation will have to possess sufficient flexibility to describe the the deformation of the wave function in the field. Such flexibility is only partially achieved with the orbital Gaussians employed in the present calculations.

The correlated Gaussians that can be considered for calculations on molecules in magnetic fields are the following functions:

$$
\phi_{k}=\exp \left[-\left(\mathbf{R}-\mathbf{s}_{k}\right)^{\prime} \mathbf{A}_{k}\left(\mathbf{R}-\mathbf{s}_{k}\right)\right]
$$

where $\mathbf{R}$ is a vector with the length of $3 N$ built from the $\mathbf{R}_{i}$ vectors, $\mathbf{s}_{k}$ is a vector containing $N$ Gaussian shift vectors, $\mathbf{A}_{k}$ is a $3 N \times 3 N$ dimensional, symmetric, positive definite matrix of Gaussian exponential factors, and prime denotes transposition of the vector. If $\mathbf{A}_{k}$ is diagonal with first three diagonal elements equal to each other, the next three elements equal to each other, and so on, and if only the $z$ coordinates of $\mathbf{s}_{k}$ are non-zero, then $\phi_{k}$ becomes equivalent to a product of orbitals, which is the type of the basis functions used in the present calculations. Thus, by allowing the A matrix to have non-zero off-diagonal matrix elements and by allowing the Gaussian centers to move away from the $z$ axis, a generalized explicitly-correlated form of the basis functions is obtained. Such functions can much more effectively describe the state of the molecule under influence of a static magnetic field. An even more general form of the correlated Gaussian can be obtained by allowing for the exponential factors to be complex numbers:

$$
\phi_{k}=\exp \left[-\left(\mathbf{R}-\mathbf{s}_{k}\right)^{\prime}\left(\mathbf{A}_{k}+\mathrm{i} \mathbf{C}_{k}\right)\left(\mathbf{R}-\mathbf{s}_{k}\right)\right]
$$

where $\mathbf{C}_{k}$ is a symmetric matrix and $\mathrm{i}=\sqrt{(-1)}$.

\section{Acknowledgments}

This work was supported by the Norwegian Research Council through the CoE Centre for Theoretical and Computational Chemistry (CTCC) Grant No. 179568/V30 and the Grant No. 171185/V30 and through the European Research Council under the European Union Seventh Framework Program through the Advanced Grant ABACUS, ERC Grant Agreement No. 267683. LA thanks CTCC for supporting his visits to Oslo. 
TABLE I: Total energies $(E)$ calculated for the HD molecule with and without the magnetic field in hartrees. The magnetic field $\mathbf{B}$ is oriented along the bond of the molecule (the $z$ axis) and has the lengths of 0.15 and 0.30 a.u. The notation $(e / p / d)$ indicates how many orbitals are used for the electrons $(e)$, for the proton $(p)$, and for the deuteron $(d)$. The energies are in a.u.

\begin{tabular}{cccc}
\hline \hline No. of orbitals $(e / p / d)$ & $E$ without field & $E$ with field 0.15 a.u. & $E$ with field 0.30 a.u. \\
\hline $6 / 1 / 1$ & -1.1037636163 & -1.0947683818 & -1.0690340166 \\
$8 / 2 / 2$ & -1.1136166947 & -1.1046585541 & -1.0791143507 \\
$10 / 3 / 3$ & -1.1174273356 & -1.1083813961 & -1.0830439178 \\
$12 / 4 / 4$ & -1.1189995965 & -1.1100736458 & -1.0845971917
\end{tabular}


TABLE II: Some expectation values calculated for the HD molecule with and without the magnetic field in hartrees using the non-BO wave functions. The field $\mathbf{B}$ is oriented along the bond of the molecule (the $z$ axis) and has the lengths of $B_{z}$ $=0.15$ a.u. and $B_{z}=0.30$ a.u. The expectation values shown include: the average distance between the deuteron and the proton, $\left(\left|\left\langle r_{d}-r_{p}\right\rangle\right|\right)$, and the squares of the average displacements of the deuteron and the proton from the $z$ axis (i.e. from the axis along which the $\mathbf{B}$ vector is not zero), $\left\langle x_{d}^{2}\right\rangle$ and $\left\langle x_{p}^{2}\right\rangle$, respectively. The notation $(e / p / d)$ indicates how many orbitals are used for the electrons $(e)$, for the proton $(p)$, and for the deuteron $(d)$. The results are in a.u.

\begin{tabular}{|c|c|c|c|c|}
\hline No. of FSGOs $(e / p / d)$ & $B_{z}$ & $\left|\left\langle r_{d}-r_{p}\right\rangle\right|$ & $\left\langle x_{d}^{2}\right\rangle$ & $\left\langle x_{p}^{2}\right\rangle$ \\
\hline \multirow[t]{3}{*}{$6 / 1 / 1$} & 0.0 & 1.4627 & 0.0043 & 0.0091 \\
\hline & 0.15 & 1.4526 & 0.0043 & 0.0090 \\
\hline & 0.30 & 1.4273 & 0.0042 & 0.0088 \\
\hline \multirow[t]{3}{*}{$8 / 2 / 2$} & 0.0 & 1.4557 & 0.0044 & 0.0095 \\
\hline & 0.15 & 1.4464 & 0.0044 & 0.0094 \\
\hline & 0.30 & 1.4224 & 0.0043 & 0.0092 \\
\hline \multirow[t]{3}{*}{$10 / 3 / 3$} & 0.0 & 1.4586 & 0.0044 & 0.0097 \\
\hline & 0.15 & 1.4442 & 0.0043 & 0.0094 \\
\hline & 0.30 & 1.4252 & 0.0043 & 0.0093 \\
\hline \multirow[t]{3}{*}{$12 / 4 / 4$} & 0.0 & 1.4606 & 0.0045 & 0.0098 \\
\hline & 0.15 & 1.4504 & 0.0044 & 0.0095 \\
\hline & 0.30 & 1.4272 & 0.0043 & 0.0093 \\
\hline
\end{tabular}


[1] H. S. Brandi, Phys. Rev. A 11, 1835 (1975).

[2] C. Schimeczek, S. Boblest, D. Meyer, and G. Wunner, Phys. Rev. A 88, 012509 (2013).

[3] G. Wunner and H. Ruder, Phys. Scr. 36, 291 (1987).

[4] H. Ruder, G. Wunner, H. Herold, and F. Geyer, SPRINGER, 1994.

[5] P. Schmelcher and L. S. Cederbaum, Phys. Rev. A 40, 3515 (1989).

[6] P. Schmelcher, L. S. Cederbaum, and H. D. Meyer, Phys. Rev. A 38, 6066 (1988).

[7] P. Schmelcher, L. S. Cederbaum, and H. D. Meyer, J. Phys. B 21, L445 (1988).

[8] T. Detmer, P. Schmelcher, and L. S. Cederbaum, J. Phys. B 28, 2903 C(1995).

[9] P. Schmelcher and L. S. Cederbaum, Int. J. Qauntum Chem. 64, 501 (1997).

[10] P. Schmelcher, T. Detmer, and L. S. Cederbaum, Phys. Rev. 64, 023410 (2001).

[11] K. K. Lange, E. I. Tellgren, M. R. Hoffmann, and T. Helgaker, Science 337, 327 (2012).

[12] W. Becken, P. Schmelcher, and F. K. Diakonos, J. Phys. B: At. Mol. Opt. Phys. 32, 1557 (1999).

[13] O.-A. Al-Hujaj and P. Schmelcher, Phys. Rev. A 70, 033411 (2004).

[14] J. E. Avron, I. W. Herbst, and B. Simon, Ann. Phys. N.Y. 114, 431 (1978).

[15] M. Vincke and D. Baye, J. Phys. B 21, 2407, (1988).

[16] P. Schmelcher and L. S. Cederbaum, Int. J. Quantum Chem. 64, 501 (1997).

[17] K. Runge and J. R. Sabin, Int. J. Quantum Chem. 64, 561 (1997).

[18] M. D. Jones, G. Ortiz, and D. M. Ceperley, Int. J. Quantum Chem. 64, 523 (1997).

[19] Y. P. Kravchenko and M. A. Liberman, Int. J. Quantum Chem. 64, 513 (1997); Phys. Rev. A 57, 1 (1998).

[20] P. Schmelcher, L. S. Cederbaum, and H. D. Meyer, J. Phys. B 21, L445 (1988).

[21] T. Detmer, P. Schmelcher, and L. S. Cederbaum, J. Phys. B 28, 2903 (1995).

[22] S. N. Datta and A. Misra J. Chem. Phys. 114, 9209 (2001).

[23] P. M. Kozlowski and L. Adamowicz, Chem. Rev. 93, 2007 (1993).

[24] S. Bubin, M. Pavanello, W.-Ch. Tung, K. L. Sharkey, and L. Adamowicz, Chem. Rev. 113, 36 (2013).

[25] J. Mitroy, S. Bubin, W. Horiuchi, Y. Suzuki, L. Adamowicz, W. Cencek, K. Szalewicz, J. Komasa, D. Blume, and K. Varga, Rev. Mod. Phys. 85, 693 (2013).

[26] M. Hoshino, H. Nishizawa, and H. Nakai, J. Chem. Phys. 135, 024111 (2011).

[27] H. Nakashima, Y. Hijikata, and H. Nakatsuji, Astrophys. J. 770, 144 (2013).

[28] M. Stanke and L. Adamowicz, J. Chem. Phys. 117, 10129 (2013). 


\section{${ }^{*}$ Graphical Abstract}

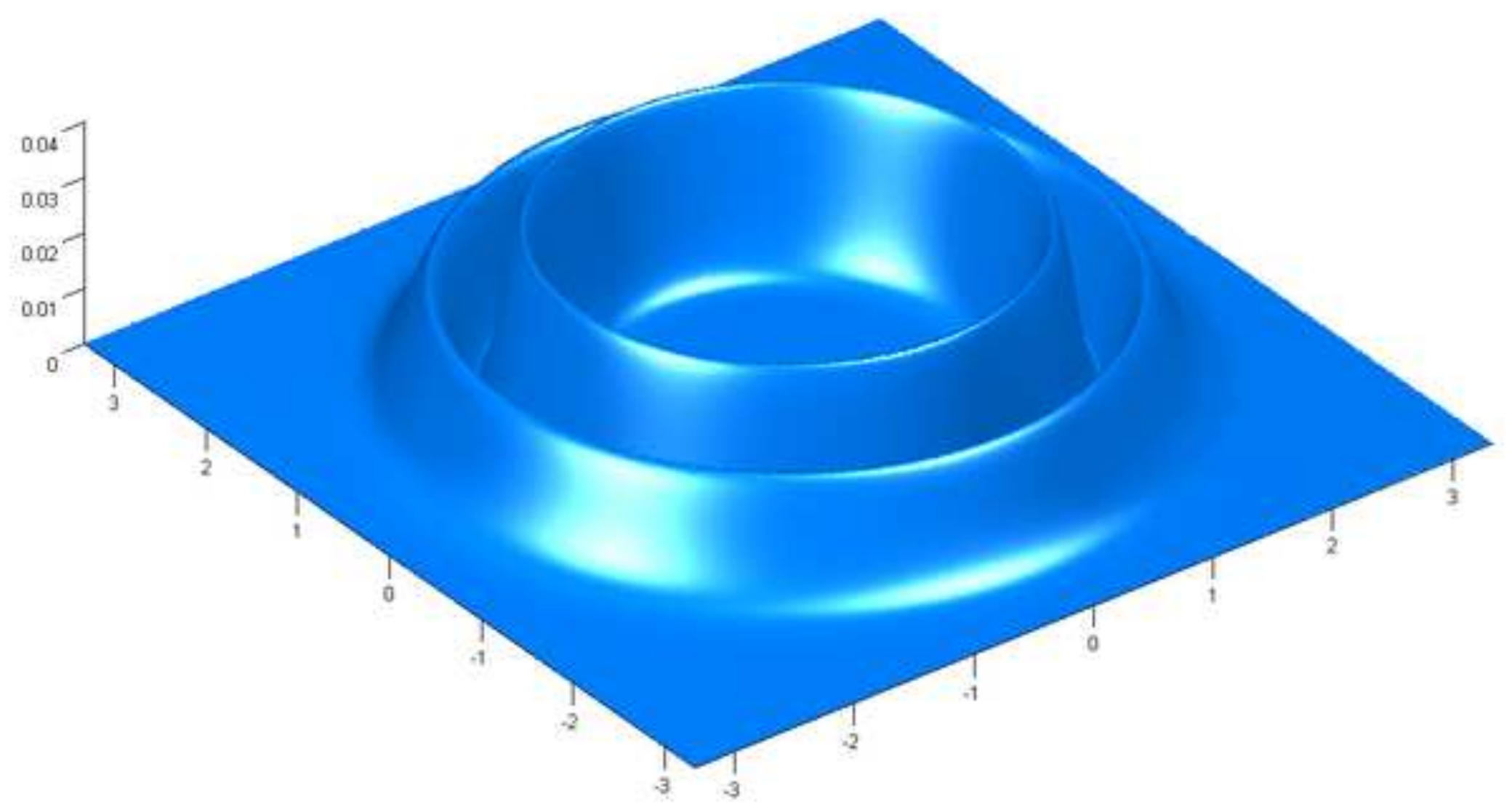

